\title{
Settlement Service Literacy and the Relationship Between Service Utilisation and Wellbeing Among Newly Arrived Migrants: A Mixed Methods Systematic Review Protocol
}

\author{
Kerry Woodward ${ }^{1 *}$, Michael Polonsky ${ }^{1}$, Julie Green $^{2}$, Julianne Abood ${ }^{3}$, \\ Andre Renzaho ${ }^{3}$ \\ ${ }^{1}$ Deakin Business School, Deakin University, 221 Burwood Hwy, Burwood VIC 3125 Australia \\ ${ }^{2}$ Raising Children Network Ltd, Parenting Research Centre, Level 5/232 Victoria Parade, East \\ Melbourne VIC 3002 \\ ${ }^{3}$ School of Social Sciences and Psychology, Western Sydney University, Locked Bag 1797, Penrith \\ 2751 NSW Australia
}

\begin{abstract}
Background: Settlement service literacy refers to the ability of migrants to access, understand and critically navigate settlement services. In Australia, increasing numbers of culturally and linguistically diverse migrants require settlement services to assist their transition. However, there are barriers to migrant's ability to utilise settlement services which are related to their level of settlement service literacy. This review aims to shed light on how settlement service literacy influences new migrant's utilisation of settlement services, and the consequences that it has for health, wellbeing and sense of belonging.
\end{abstract}

Methods: The review will follow the guidelines laid out by the Preferred Reporting Items for Systematic Reviews and Meta-Analyses (PRISMA). Literature searches will be undertaken in CINAHL, EMBASE, PubMed, PsycINFO, Scopus, Web of Science, Sociological Abstracts, SocIndex, ProQuest Social Science Database, and Google Scholar. Grey literature and relevant government bodies, non-government organisations, service providers and research institutes will also be searched. Studies included will report primary data (qualitative and quantitative) on new migrant's (under five years) ability to utilise settlement services in high income countries. Studies that meet the inclusion criteria will be imported to Covidence, two researchers will screen the studies in a two part process (title and introduction scan; and full text) for relevance. Data extracted will include general publication information (author, country, year, and publication), type of study, participants, type of settlement service, measured outcomes, and the study aims, methods and results. Finally, data will be synthesised using a narrative approach.

Discussion: The review will provide insight into the relationship between settlement service literacy and service utilisation and wellbeing for new migrants. The review will also provide data to inform settlement service policy to better cater for the needs of migrants.

\footnotetext{
* Correspondence concerning this article should be addressed to Kerry Woodward, Deakin Business School, Deakin University, 221 Burwood Hwy, Burwood VIC 3125 Australia. Email: kerry.woodward@deakin.edu.au
} 
Systematic review registration: This protocol has been submitted to the international prospective register of systematic reviews and meta-analysis (PROSPERO) and is currently under review.

Keywords: Settlement service literacy, service utilisation, new migrants, wellbeing

\section{Background}

\subsection{Settlement service literacy}

Australia is one of the most multi-faith and culturally and linguistically diverse (CALD) societies in the world (ABS, 2013). Increasing numbers of CALD migrants has required the Australian government to establish a wide range of settlement services to facilitate their adjustment to the new environment. Successful engagement with settlement services provides migrants a pivotal sense of identity or 'sense of belonging' in Australia (Renzaho et al., 2011; Renzaho, McCabe \& Sainsbury, 2010). Whilst government policy enshrines migrants' rights to settlement services, underutilisation of settlement services occurs due to a range of issues related to service provision, community attitudes, language, availability of interpreters, migrants' service awareness, and perceptions of discrimination, as well as financial barriers (Renzaho et al., 2011; Renzaho, McCabe \& Sainsbury, 2010). It is well documented that many migrants have challenging settlement experiences as a result of poor settlement service literacy (SSL) and structural inequity (SCOA, 2015).

SSL refers to the extent to which migrants have foundational information, knowledge, and skills to access and effectively utilise settlement services; specific developmental processes by which these skills can be developed; competencies to critically navigate settlement services; and political skills to mobilize mainstream society so settlement services can be prioritised on political agendas (Masinda, 2014). Masinda (2014) provides a useful typology of SSL consisting of three key threads: basic settlement service literacy, critical settlement service literacy, and political settlement service literacy. These categories cover migrant's ability to access and be aware of relevant settlement services (basic), their ability to question and challenge services and service providers (critical), and their ability to shape and influence the design and implementation of settlement services (political).

The development of competencies that enable CALD migrant communities to interact effectively with available services and support can contribute to reducing structural inequity, increase sense of belonging, and promote wellbeing. SSL involves a wide range of interactive processes involved in everyday information exchange, such processes are central to making critical judgements and decisions in settlement service settings (Masinda, 2014). In this way, SSL extends beyond technical and functional skills for coping with the demands of everyday life and work in a knowledge economy (ABS, 2013b). SSL constructs literacy as part of the infrastructure used to promote healthy lifestyles; reveals the ways in which it is patterned by history, social and cultural life, values and relationships; and how it contributes to socioeconomic development, enhancing individuals' capacity for social awareness and critical reflection as a basis for personal and social change (Masinda, 2014). SSL is closely linked to the individual's overall literacy level, which is lower among Australians whose first language is not English (ABS, 2013b).

\subsection{Aim of the review}

The aim of this review is to map out and assess research that provides insights into the relationship between SSL and service utilisation for new migrants. Understanding the level of SSL among new migrants has important implications for the integration and sense of

Social Science Protocols, February 2020, 1-7.

http://dx.doi.org/10.7565/ssp.2020.2801 
belonging for new migrants, as well as for reducing structural inequalities migrants face in their new country. Lack of SSL among migrants forms substantial barriers to job seeking, education options, and accessing settlement services due to a lack of communication confidence (limiting help-seeking). Moreover, low SSL can contribute to perceived limitations of and deficiencies in interpreting services, limited computer skills, and a negative view of available services (e.g. migrants' inability to resolve issues with service providers due to insufficient language and service knowledge or navigation skills). Importantly, overall wellbeing, health, and education outcomes may be enhanced by reducing structural inequalities and increasing new migrants' access to and control over settlement services.

\subsection{Review question}

The question guiding the review is: how does SSL impact on service utilisation and wellbeing among newly arrived migrants?

\section{Methods/Design}

\subsection{Study design}

The review will follow the guidelines laid out by the Preferred Reporting Items for Systematic Reviews and Meta-Analyses (PRISMA) and employ the PRISMA flowchart.

\subsection{Participants}

The review will include studies of new migrants (within five years), both voluntary (economic) and involuntary (refugees and asylum seekers) living in high income countries. This review will largel focus on cross-sectional studies, as such no comparator will be included.

\subsection{Intervention design and focus}

The review will include studies that report primary data and investigate the uptake, accessibility or outcomes of settlement services for new migrants in high income countries and offer some insight into settlement service literacy. Importantly, as SSL is a relatively new and little used term, the review will look for indicators of SSL that are not specifically termed SSL, such as migrant's knowledge or awareness of available services, ability to question service providers, ability to influence the design of services, or their ability to mobilise political support for services. The review will include any studies, both qualitative and quantitative, that focus on new migrant's settlement service literacy and new migrant's ability to utilise services and wellbeing. Further, the review will include grey literature, as well as evaluations and reports from government, non-government organisations, research institutes, transnational institutions, and service providers. Settlement services cover a broad remit including: health, counselling and therapy; education, literacy and language; orientation, leisure and recreation services; financial and housing services; legal and justice services; and translation and interpreting. Studies included in the review must focus on one or more of these services, as well as refer to settlement service literacy. The review will include studies in English only, due to imitated resources. Studies between January 2000 and January 2020 will be considered for review.

\subsection{Outcome of interest}

The review aims to shed light on the relationship between settlement service literacy and utilisation of settlement services and migrant wellbeing. For instance, how does the level of 
SSL (i.e basic, critical, or political) affect migrant's ability to know about, use, question, influence, and design settlement services.

\subsection{Search strategy}

Key search terms were developed via consultation between the research team, and from a cursory review of settlement service literature, a number of search terms were derived from Lim et al.'s (2005) exploration of the range of settlement services available in the Greater Toronto area in Canada.

Preliminary searches were undertaken on $19^{\text {th }}$ September 2019 , to test and further develop appropriate keywords, MeSH words and truncations (*). Keywords will be linked using appropriate Boolean operators. The search terms to be used are:

(migrant* OR immigrant* OR refuge* OR asylum seeker* OR CALD or "culturally and linguistically diverse" OR newcomer)

AND

("settlement service literacy" OR "service literacy" OR literacy OR health OR "health literacy" OR "health program*" OR medical OR education OR training OR literacy OR school* OR legal OR justice OR language OR ESL or "English as a second language" OR advocacy OR counsel* OR therapy OR "food service*" OR "emergency food service*" OR form filling OR information service* OR referral OR childcare OR orientation OR recreation OR leisure OR translation OR interpretation)

AND

(uptake OR utili?ation OR access* OR outcome* OR participat*)

These search terms cover the three key threads of the review question; new migrants; settlement service or settlement service literacy; and, settlement service utilisation or accessibility.

\subsection{Information sources}

The review will involve a comprehensive search of the following databases:

1. CINAHL

2. EMBASE

3. PubMed

4. PsycINFO

5. Scopus

6. Web of Science

7. SocIndex

8. ProQuest Social Science Database

9. Google Scholar

In addition to searching peer reviewed literature, a search of grey literature will be conducted. Including key institutions, organisations and government websites and publications:

Department of Foreign Affairs and Trade, Australian Government; Department of Social Services, Australian Government; Australian Institute of Family Studies; Settlement Services International; AMES Australia; Refugee Council of Australia; Diversitat Australia; Victorian Refugee Health Network; United Nations High Commission for Refugees; International Organisation for Migration; World Health Organisation. 
The above organisations and government departments have been chosen as they advocate for, design and/or implement settlement services, but also undertake research or produce reports on settlement services. Service providers or advocacy organisations that do not undertake research or produce reports will be excluded.

The search will also include reference lists of included studies; forward and backward citation searching in both Google Scholar and Web of Science, as well as consultation and recommendations from experts in the field.

\subsection{Data collection}

The search results will be imported to Covidence and duplicates will be removed. Titles and abstracts will be assessed for relevance to the review and to ensure studies meet the inclusion criteria. Finally, the full text of studies deemed relevant for the review in the second stage will be read and assessed for relevance/eligibility. The screening process will be undertaken by two researchers.

\subsection{Data extraction}

Data extraction will be based on a modification of the Cochrane Public Health Group Data Extraction and Assessment Template. Information to be extracted will include: general publication information (author, country, year, and publication), type of study, participants, type of settlement service, measured outcomes, and the study aims, methods and results.

\subsection{Quality assessment}

Quality assessment will be undertaken independently by two researchers. Quality assessment will be performed using the Critical Appraisal Skills Programme (CASP, 2017) for all observational studies, including qualitative and quantitative studies; Mixed-Methods Appraisal tool (MMAT) (Hong et al., 2018) will be employed to assess mixed-methods studies; and grey literature will be appraised using the AACODS tool (Tyndall, 2010) which focuses on reviews' authority, accuracy, coverage, objectivity, date, and significance.

All studies will be appraised as having a high, medium or low quality and the overall quality of the body of evidence will be assessed using the Grading of Recommendations Assessment, Development and Evaluation (GRADE) approach (Guyatt et al., 2011).

\subsection{Data synthesis}

As the included studies are likely to employ diverse methodologies, data will be synthesised using narrative synthesis. Narrative synthesis is useful in combining diverse types of evidence - which we expect the search to yield, and making heterogeneity between studies transparent (Barnett-Page \& Thomas, 2009). Drawing from Popay et al. (2006), the narrative synthesis will proceed through 4 steps: develop a theory of how SSL influences service utilisation; undertake a preliminary synthesis of findings of included studies; explore the relationship in the data; and assess the robustness of the synthesis.

\section{Discussion}

The review will provide insight into the relationship between settlement service literacy and service utilisation and wellbeing for new migrants; and will provide data to inform settlement service policy to better cater for the needs of migrants. The systematic review will provide data that sheds light on how aspects that enhance the effectiveness of SSL, including migrants' ability to change the dynamics of SSL to better cater for their needs, to criticise providers, and to contribute to the development or implementation of programmes. Given that Social Science Protocols, February 2020, 1-7. 
the concept of settlement service literacy may not be widely used, it is possible that most of studies will be located and synthesised using proxies for SSL.

\section{List of Abbreviations}

SSL - Settlement service literacy

\section{Declarations}

Competing interests: The authors declare that they have no competing interests.

Funding: This research is funded by the Australian Research Council.

\section{References}

Australian Bureau of Statistics. (2013). Australian Social Trends, Cat No. 4102.0. ABS, Canberra.

Australian Bureau of Statistics. (2013b). Programme for the International Assessment of Adult Competencies, Australia, 2011-12, Cat No. 4228.0. ABS, Canberra.

Barnett-Page, E., \& Thomas, J. (2009). Methods for the synthesis of qualitative research: a critical review. BMC Medical Research Methodology, 59(1).

Critical Appraisal Skills Programme (CASP). (2017). Retrieved from https://caspuk.net/casp-tools-checklists/.

Guyatt, G., Oxman, A.D., Akl, E.A., Kunz, R., Vist, G., Brozek, J., Norris, S., ... Schünemann H. (2011). GRADE guidelines: 1. Introduction-GRADE evidence profiles and summary of findings tables. Journal of Clinical Epidemiology, 64(4), 383-394.

Hong, QN., Pluye, P., Fàbregues, S., Bartlett, G., Boardman, F., Cargo, M., Dagenais, P., ... O'Cathain, A. (2018). Mixed methods appraisal tool (MMAT). Retrieved from $\mathrm{http} / / /$ mixedmethodsappraisaltoolpublic.pbworks.com/w/file/fetch/127916259/MMAT_20 18_criteria-manual_2018-08-01_ENG.pdf.

Lim, A., Lo, L., Siemiatycki, M., \& Doucet, M. (2005). Newcomer services in the Greater Toronto area: an exploration of the range and funding sources of settlement services. Geography Publications and Research, 41. Retrieved from https://digital.library.ryerson.ca/islandora/object/RULA\%3A227.

Masinda, M.T. (2014) Immigrant Settlement Services Literacy. International Journal of Social Work, 1(2), 1-13.

Popay, J., Roberts, H., Sowden, A., Petticrew, M., Arai, L., Rodgers, M., Britten, N., ... Duffy, S. (2006). Guidance on the conduct of narrative synthesis in systematic reviews: A product from the ESRC Methods Programme. Lancaster University. Retrieved from http://citeseerx.ist.psu.edu/viewdoc/download?doi=10.1.1.178.3100\&rep=rep1\&type=pdf.

Renzaho, A.M.N., Green, J., Mellor, D., \& Swinburn, B. (2011). Parenting, family functioning and lifestyle in a new culture: the case of African migrants in Melbourne, Victoria, Australia. Child \& Family Social Work, 16(2), 228-240.

Renzaho, A.M.N., McCabe, M., \& Sainsbury, W.J. (2011). Parenting, role reversals and the preservation of cultural values among Arabic speaking migrant families in Melbourne, Australia. International Journal of Intercultural Relations, 35(4), 416-424.

Settlement Council of Australia. (2015). National Settlement Services Outcomes Standards. SCOA: Sydney. Retrieved from http://scoa.org.au/policy-hub/scoa-publications/nationalsettlement-services-outcomes-standards/. 
Tyndall, J. (2018) AACODS checklist. Flinders University. Retrieved from https://dspace.flinders.edu.au/xmlui/bitstream/handle/2328/3326/AACODS_Checklist.pdf. Wali, N., Georgeou, N., \& Renzaho, A.M.N. (2018). 'Life Is Pulled Back by Such Things': Intersections Between Language Acquisition, Qualifications, Employment and Access to Settlement Services Among Migrants in Western Sydney. Journal of Intercultural Studies, 39(1), 85-101. 